\title{
Detection of reinforcement bars in concrete slabs by infrared thermography and microwaves excitation
}

\author{
by F. Brachelet*, S. Keo*, D. Defer* and F. Breaban*
}

\author{
* Laboratoire de Génie Civil et Géo-Environnement, Université Lille Nord de France \\ Université d'Artois, Technoparc Futura, 62400 Béthune, France. \\ franck.brachelet@univ-artois.fr
}

\begin{abstract}
This paper presents a NDT method by infrared thermography with a microwave excitation system applied to the detection of metallic parts: a metal ring behind a wooden plate of $1 \mathrm{~cm}$ thick, a reinforcement bar in front of a concrete wall and the detection of reinforcement bars equally spaced in a concrete slab of $6.5 \mathrm{~cm}$ thick. The excitation device is based on a magnetron associated with a horn pyramidal antenna. A contrast algorithm applied to the sequences of thermograms highlights metal ring or bars location.
\end{abstract}

\section{Introduction}

Many methods of structural inspection are based on the propagation of electromagnetic waves and are able to provide qualitative and quantitative information on the internal structure of the inspected elements [1]. Among the existing techniques such as radiography, gammagraphy or radar applied to civil engineering, active infrared thermography often requires relatively long test duration because of high time constant of thermal diffusion within the inspected elements [2]. Moreover, thermal stimulations applied from the external surface limit the auscultation depth or impose long-term tests. This paper aims to present the development of a new active infrared thermography method based on a microwave excitation system [3]. Compared to other sources traditionally used in active infrared thermography, the microwaves can propagate into the volume of a concrete element. The waves undergo the absorption, the reflection and the diffraction effects while they impact any metal parts [4,5]. This new method has been applied to defects detection in the case of a metal element placed behind a wooden plate of $1 \mathrm{~cm}$ thick, and more particularly to vertical reinforcement bars in a concrete wall where radiothermal interference phenomena can be observed.

\section{Excitation and detection device}

A microwave source was set up based on a commercial magnetron of $800 \mathrm{~W}$ at a $2.45 \mathrm{GHz}$ frequency associated to a pyramidal horn antenna with aperture dimensions of $A=59 \mathrm{~cm}$ and $B=56 \mathrm{~cm}$. As shown in figure 1 a., the generator was adapted to a horn antenna which serves to guide the microwave beam onto the tested samples. This system is placed on a tripod to ease the antenna orientation. Figure 1.b shows the radiation pattern for the horn antenna providing an aperture around $40^{\circ}$ in the sagittal and azimuthal plans. Considering all the safety, a protective enclosure was built in order to protect operators from the radiation. Thermograms are recorded with a CEDIP Silver 420 cooled camera equipped with a $320 \times 240$ pixels InSb detector. The camera placed at a distance of $1.5 \mathrm{~m}$ far from the system, provides a field of view of the heated area. The thermograms are recorded at a frame rate of $1 \mathrm{~Hz}$ during the tests.
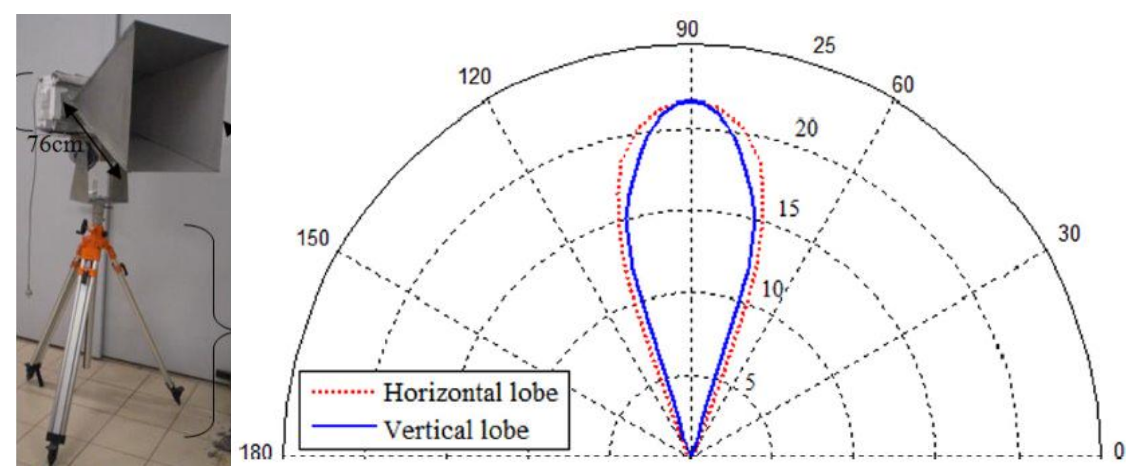

Fig. 1 a\&b. Microwaves horn antenna and the resulting radiation pattern diagram (principal lobes) 


\section{Data analysis procedure}

The method used to analyze thermogram series is based on a contrast algorithm. The non-uniformity of the beam, caused by its reflection from the specimen with the antenna, generates an inhomogeneous volumetric heating in the sample and may lead to misinterpret raw thermograms [6]. The amplitude of this non-uniformity also depends on the detected material nature. Thus, the tests are conducted in two stages. The calculation principle is to subtract first thermograms series recorded on a specimen without defect with a series recorded on a specimen containing some inclusions. This subtraction is carried out on each point and at the same times. This approach can reduce the nonuniformity effect of the excitation. The initial temperature value is then subtracted from the resulting contrast sequence, which allowed to consider only the temperature rise in case of the temperatures of the two specimens would not be identical.

The method used to analyze the series of thermograms is based on a contrast algorithm. The non-uniformity of the microwaves beam, caused by its reflection from the specimen with the antenna, produced an inhomogeneous heat generated in the sample that can lead to misinterpretations of thermographic images. The amplitude of this nonuniformity depends on the detected material nature too. Thus, the tests are conducted in two stages. The calculation principle is shown in figure 2 .

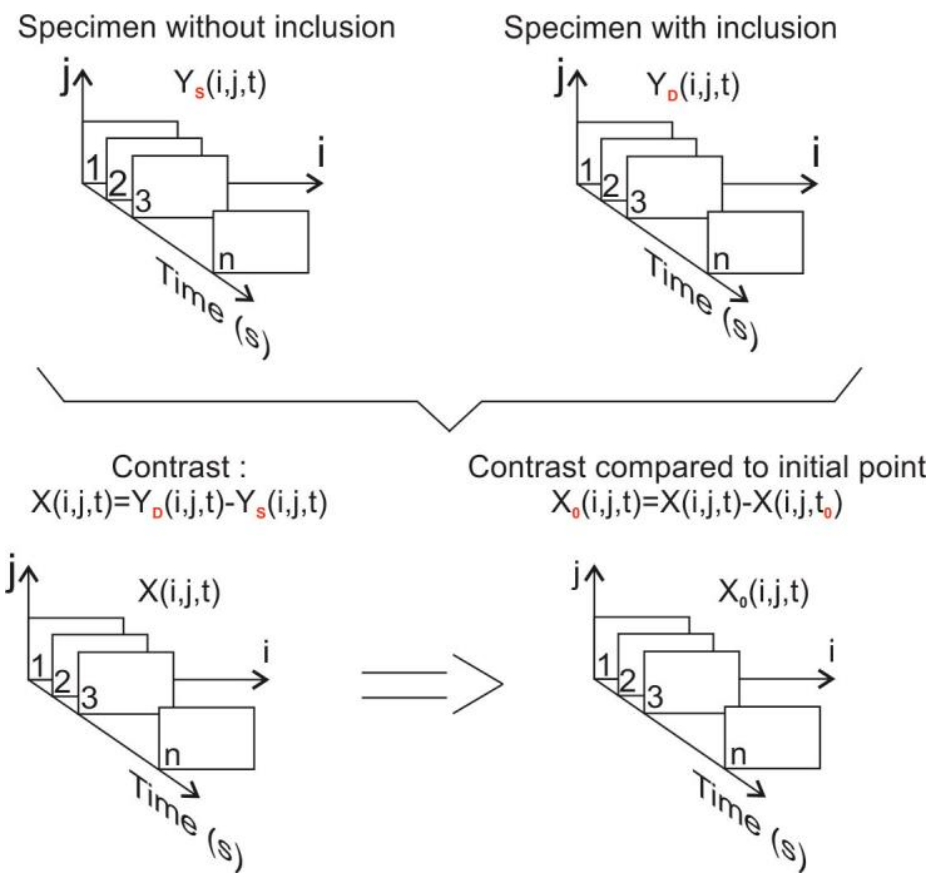

Fig. 2. Thermograms analysis principle

Considering the same point $Y(\mathrm{i}, \mathrm{j}, \mathrm{t})$ on the tested specimens surface, the difference $(\mathrm{X})$ was carried out at the same instants between the temporal evolution of a thermographic sequence of a specimen considered as being without defect $\left(Y_{S}\right)$ and a specimen containing inclusions $\left(Y_{D}\right)$. This approach can reduce the non-uniformity effect of the excitation. The initial temperature value was then subtracted from the contrast sequence, which allowed to consider only the temperature rise $\left(X_{0}\right)$ in case the temperatures of the two specimens would not be identical. This algorithm was applied to each point (each $\mathrm{i}, \mathrm{j}$ ) comprising the thermogram.

\section{Results and discussion}

\subsection{Metal ring behind a wooden plate}

The first test is applied to a metal ring placed behind a wooden plate. The experimental set-up is shown in figure 3. The wood is heated under the effect of the microwaves through the residual water contained in the fibers. The metal insert positioned in rear face allows waves to be reflected and then to be reabsorbed by the water contained in the wood, which leads to a rise in temperature higher than on the area without insert. Diffraction effects are also visible on the thermogram. They are materialized by a circular circle with higher temperature on the surrounding area. This test shows the interest of microwaves excitation because it reveals the presence of the metal piece behind the plate of $1 \mathrm{~cm}$ 
thick. The results were obtained after $100 \mathrm{~s}$. The raising of the temperature is remained low $\left(<1^{\circ} \mathrm{C}\right)$, and did not damaged the sample.

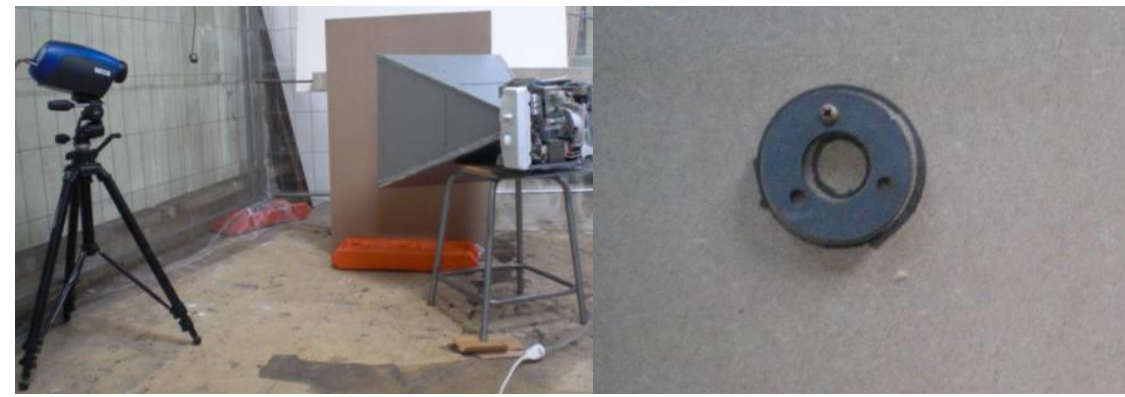

Fig. 3. Experimental set-up

The wood sample was heated for 180 seconds; thermograms shown below in figure 4 were taken at the initial time. They do not reveal the presence of a defect, but only the presence of a temperature gradient between the top and bottom of the plate.

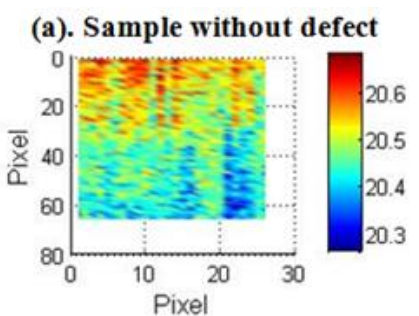

(c). Contrast of both samples

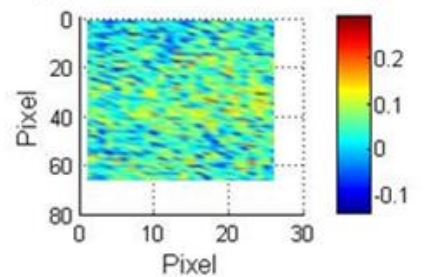

(b). Sample with defect

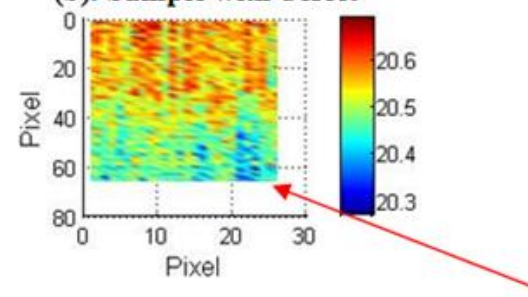

(d). Contrast compared to initial instant

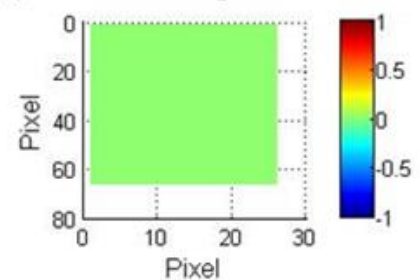

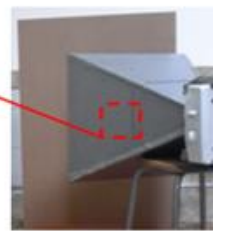

Fig. 1. Thermograms of wood plate at the initial time

The contrast function below shows that it is interesting to place at $t=170$ s to get maximum contrast.

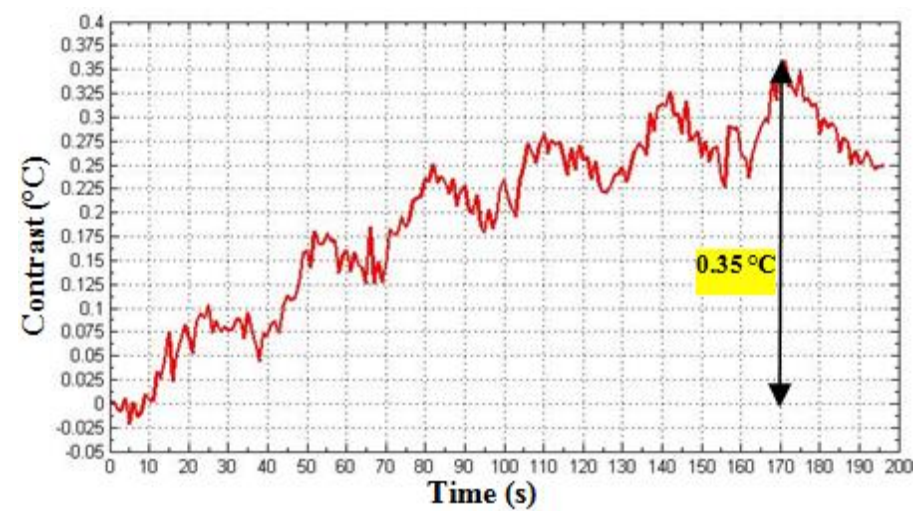

Fig. 5. Contrast function for the wood plate

The first test on a wooden plate without defect shows a moderate increase in temperature $\left(0.7^{\circ} \mathrm{C}\right)$. The wood is heated up under the effect of the microwaves thanks to the residual water contained in the fibers. The second test is performed by placing this time a metallic insert against the rear face of the sample. The incoming waves are reflected at this location and are reabsorbed by the water contained in the wood. This leads to a rise in temperature higher than in the areas without insert. The thermograms obtained at $t=170$ s (figure 6 ) show the presence of the metal part. The difference in average temperature between the insert and the area with a sound area is of about $0.35^{\circ} \mathrm{C}$. 
(a). Sample without defect

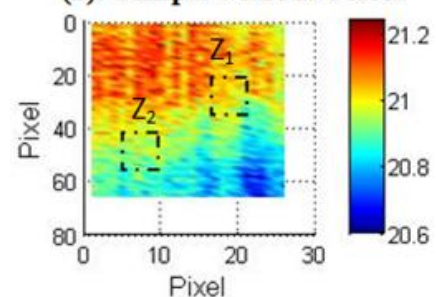

(c). Contrast of both samples

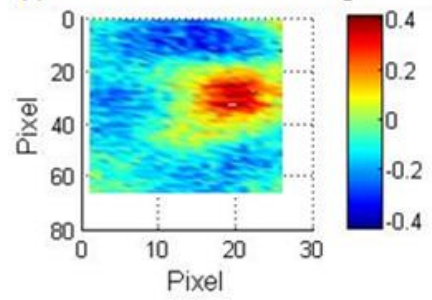

(b). Sample with defect

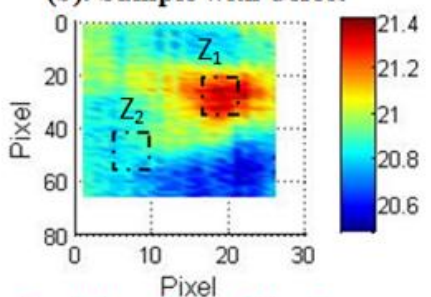

Position of metallic element

(d). Contrast compared to initial instant

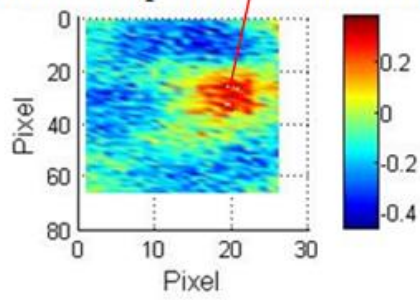

Fig. 6. Thermograms of wood plate at $t=170 \mathrm{~s}$
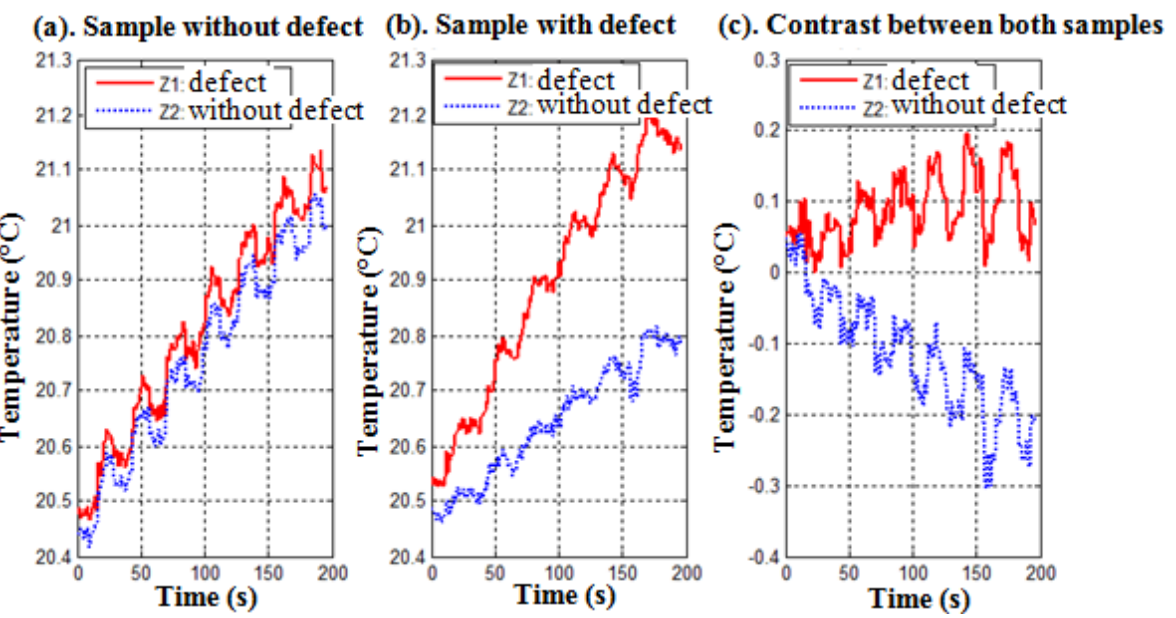

Fig. 7. Temporal contrasts for $Z_{1}$ and $Z_{2}$ areas

In order to compare evolution of temperature in the same places, two zones $Z_{1}$ and $Z_{2}$ are defined. They are located in the same places on each sample. The figure $7 \mathrm{a} \& \mathrm{~b}$ shows the evolution of temperature on wood samples with and without metal insert for both areas. Their changes are phase modulated at the rate of operation of the magnetron.

\subsection{Case of a metallic reinforcement bar}

To further the understanding of the diffraction effects created by a small object, a rebar $(\varnothing 12 \mathrm{~mm})$ is placed vertically in front of a concrete wall which does not contain any armature. The antenna is placed at $40 \mathrm{~cm}$ from the wall with an inclination of $45^{\circ}$ and the thermograms are recorded from the rear face. The results show two heated areas on both sides of the bar. This illustrates the penetration of the waves in the concrete wall and their impact on the temperature field of the rear face. 


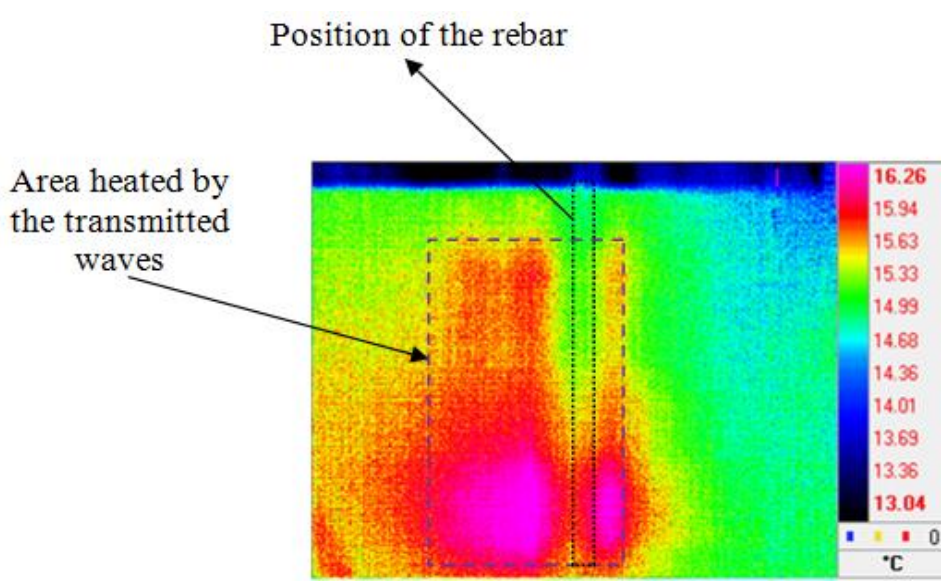

Fig. 8. Thermogram with a single reinforcement bar

\subsection{Detection of a reinforcement system in a concrete wall}

In this test, the antenna is placed at $40 \mathrm{~cm}$ from a reinforced wall with an inclination of $45^{\circ}$ relative to normal. The camera is placed on the same side with an inclination of $30^{\circ}$ in the opposite direction. The wall is $6.5 \mathrm{~cm}$ thick and has a surface of $1 \mathrm{~m}^{2}$. The average incident power is $600 \mathrm{~W}$ and the test lasts 5 minutes. This procedure is reiterated 2 times according to the contrast algorithm procedure. Figure 9 shows the results. The upper thermograms are respectively recorded from the concrete slabs containing no armature and the reinforced one at $t=250 \mathrm{~s}$. The figure $9 \mathrm{c}$ results from the subtraction between images $a$ and $b$. The figure.9d comes from the subtraction of figure $9 \mathrm{c}$ and the thermogram recorded at $\mathrm{t}=0 \mathrm{~s}$. These interference phenomena appear on the surface and generate, by the radiothermal effect, the temperature gradients observed by infrared thermography.

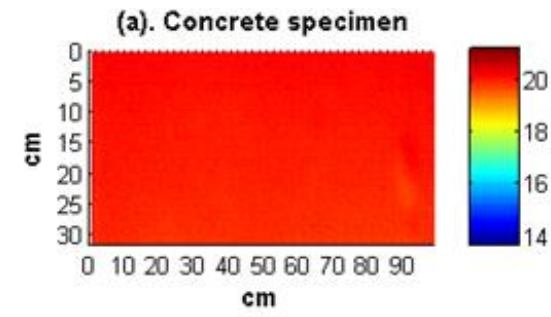

(c). Thermal contrast between the specimens

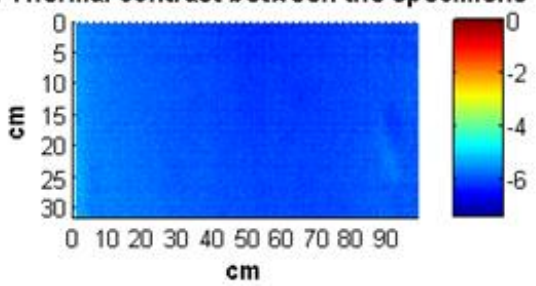

(b). Reinforced concrete specimen

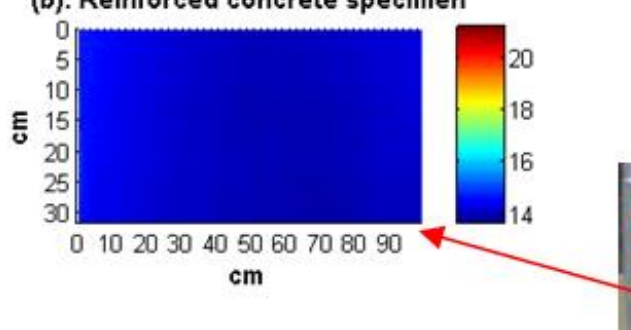

(d). Thermal contrast compared to initial instant

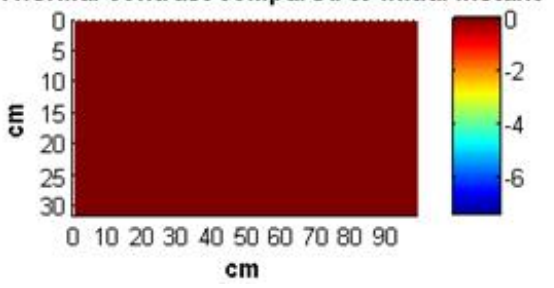

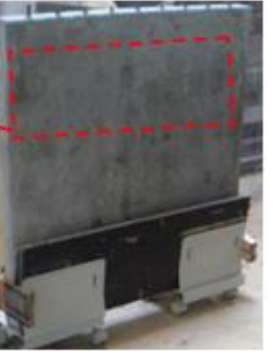

Be.

Fig. 9. Thermograms of concrete slab at the initial time

Applying the algorithm contrast at all points on the surface of the concrete walls and reinforced concrete $(\mathrm{a} 31 \mathrm{~cm} \times$ $98 \mathrm{~cm}$ area was observed), the thermograms at the initial time are shown in figure 9 . We observed a low temperature gradient to the surface of each test piece at the initial time, but there is a difference in temperature of approximately $5{ }^{\circ} \mathrm{C}$ between the two pieces (figure $9 \mathrm{a \& b}$ ) due to changes of the environmental test conditions, which means that it is necessary to observe the contrast at the initial time and subtract contrasts at every moment before estimating visualization. 
(a). Concrete specimen

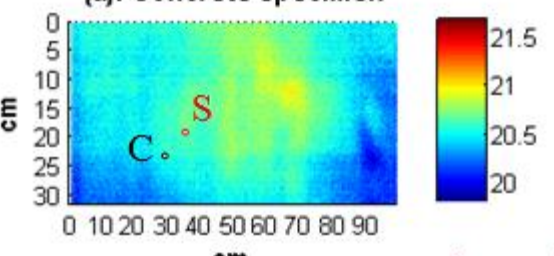

(b). Reinforced concrete specimen

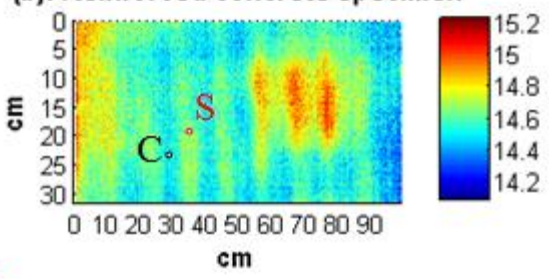

(c). Thermal contrast between the specimens
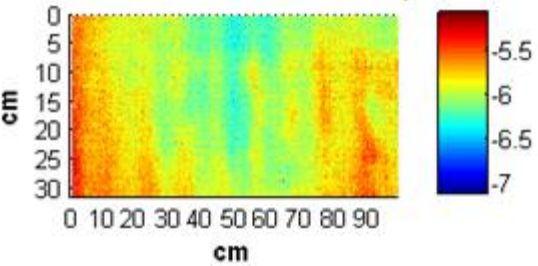

(d). Thermal contrast compared to initial instant
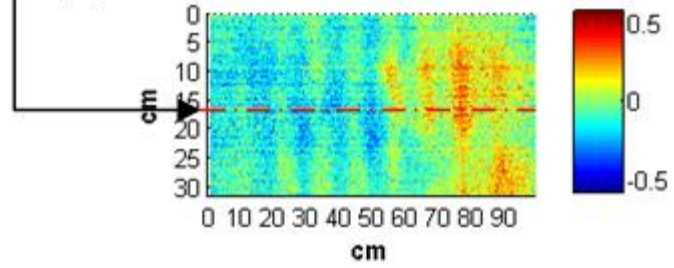

Fig. 10. Thermograms of reinforced concrete slab at $t=250 \mathrm{~s}$

Figure 10 shows the thermograms at time $250 \mathrm{~s}$. The contrast algorithm proves the presence of reinforcements in the concrete wall. In order to clarify which parts are frames, a horizontal profile along the thermal contrast was performed.

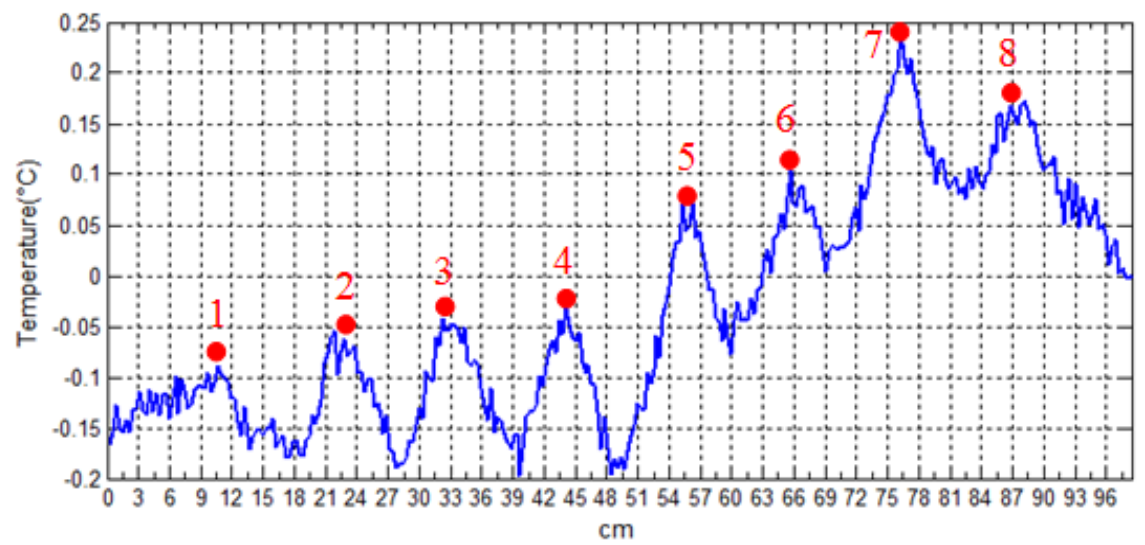

Fig. 11. Horizontal profile on the contrast image at $t=250 \mathrm{~s}$

In figure 11 , the temperature profile curve has eight points corresponding to maximum. These points correspond to the positions of frames in the wall under the diffusion microwave. The higher temperatures at the locations of reinforcements are caused by the microwave energy reflected back and dissipated between the front surface and the location of the concrete reinforcement (coating); the heat transfer mechanism in a reinforced concrete slab subjected to microwaves was explained theoretically and numerically in [7] and [8] since the 1990s. Though all frames are the same ( $3.8 \mathrm{~cm}$ from the area), the temperature profile of peaks do not have the same values of temperature. The frames \# 5,6 , 7 , and 8 are in the vicinity of the aperture of the antenna (in the direction of $45^{\circ}$ relative to the axis of the test piece) receive more energy and therefore have higher temperature than the number frames $1,2,3$, and 4 which are located further from the antenna. The ninth frame is invisible in the thermograms because it was not exposed to microwaves. The aperture of the antenna of $56 \mathrm{~cm} \times 59 \mathrm{~cm}$ cannot heat the sample over the entire width of $1 \mathrm{~m}$.

The analysis of the contrast function to determine a time at which the thermogram exhibits a maximum contrast is visible in figure 12. It represents the contrast between the steel reinforcement area and the concrete area. It shows that a maximum of contrast of $0.25^{\circ} \mathrm{C}$ is reached at time $\mathrm{t}=250 \mathrm{~s}$. 


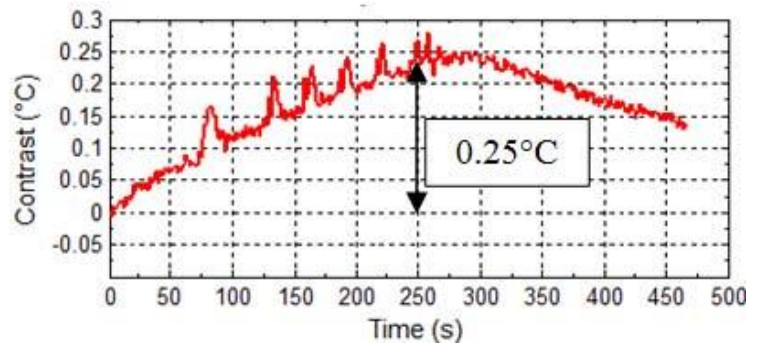

Fig. 12. Temporal contrast between the steel reinforcement area and the concrete area.

Figure 13 shows the development of two points located in the region of the frame $(S)$ and in the region of the concrete (C) of the two pieces of concrete and reinforced concrete (" $\mathrm{S}$ " and " $\mathrm{C}$ " in figure 10 a\&b). The samples were heated for a total duration of $300 \mathrm{~s}$. For the concrete wall, the two curves (figure 13a) have a same increase in temperature of $0.75^{\circ} \mathrm{C}$, elevation of $20.05^{\circ} \mathrm{C}$ to $20.80^{\circ} \mathrm{C}$. The curves are also modulated by the phases of magnetron heating as in the preceding case on the wooden plate. At the end of the heating period, there is also a relaxation step.
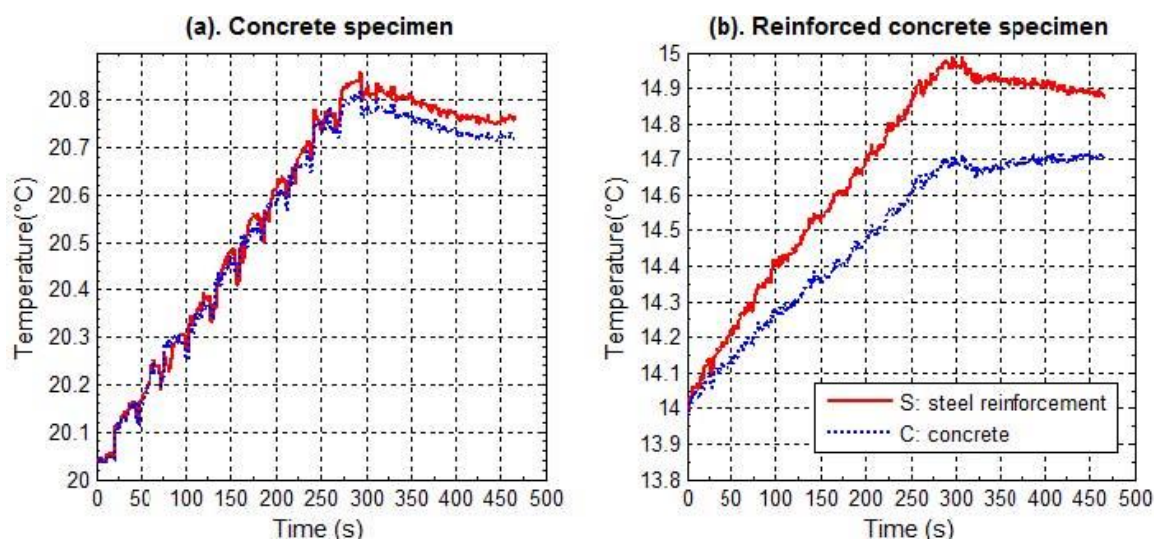

Fig. 13 a\&b. Temperature evolution of 2 point in the concrete area and in the reinforced area

The test on reinforced concrete specimen shows a difference of temperature changes, figure $13 \mathrm{~b}$. There is a temperature increase of $1^{\circ} \mathrm{C}\left(14^{\circ} \mathrm{C}\right.$ to $\left.15^{\circ} \mathrm{C}\right)$ in the vicinity of the rebar and $0.7^{\circ} \mathrm{C}\left(14^{\circ} \mathrm{C}\right.$ to $\left.14.7^{\circ} \mathrm{C}\right)$ in the concrete area.

\section{Conclusion}

The microwave excitation was applied to the detection of a metal part in a wooden plate and in a reinforced concrete. Qualitative results occurred rapidly in spite of the relative thickness of the inspected elements. In its actual configuration, the antenna allows to inspect a surface of one square meter and a protective enclosure is needed to preserve operators from microwaves effects. The contrast algorithm requires having a reference sample in order to get rid of the non-uniformity of the microwaves beam. Further works need to investigate more on the effects of interference and diffraction phenomena on the reinforcement bar layer by this infrared thermography method.

\section{REFERENCES}

[1] Breysse D., Abraham O., "Méthodologie d'évaluation non destructive de l'état d'altération des ouvrages en béton". ISBN: 2-85978-405-5, 2005, Presse des ponts et chaussées, 2005

[2] Maldague X., "Nondestructive Evaluation of Materials by Infrared Thermography", Ed. Springer London Ltd, ISBN: 1-44711-997-5, 2013

[3] S. Keo, D. Defer, F. Breaban, F. Brachelet, "Comparison between Microwave Infrared Thermography and CO2 Laser Infrared Thermography in Defect Detection in Applications with CFRP", Materials Sciences and Applications, 2013, 4, 600-605 
[4] Levesque P., Deom A., Balageas D., "Non-destructive evaluation of absorbing materials using microwave stimulated infrared thermography", proceeding QIRT Conference 1992.

[5] Balageas D., Levesque P., "EMIR: a photothermal tool for electromagnetic phenomena characterization", Revue Générale de Thermique 01/1998; DOI: 10.1016/S0035-3159(98)80050-0.

[6] S. Keo, F. Brachelet, F. Breaban, D. Defer, "Development of an Infrared Thermography Method with CO2 Laser Excitation, Applied to Defect Detection in CFRP", WASET, Int. Journal of Physical, Nuclear Science and Engineering Vol:7 No:8, 201, 2013

[7] L. E. Lagos, W. Li, M. A. Ebadian, T. L. White, R. G. Grubb, D. Foster, "Heat transfer within a concrete slab with a finite microwave heating source," International Journal of Heat and Mass Transfer, vol. 38, pp. 887-897, 1995.

[8] Zdenek P. Bazant, F. ASCE and Goangseup Z., "Decontamination of radionucleides from concrete by microwave heating. I: Theory",Engineering Mechanics - ASCE, vol.129, pp. 777-784, 2003. 